Wijnhoven, T.; Deconinck, G., "Flexible fault current contribution with inverter interfaced distributed generation," in IEEE Power and Energy Society General Meeting (PES), Vancouver, BC, Canada, 21-25 July 2013, 5 p.

doi: 10.1109/PESMG.2013.6672690

URL: http://ieeexplore.ieee.org/stamp/stamp.jsp?tp=\&arnumber=6672690\&isnu $\underline{\mathrm{mber}}=6672065$

(c) 2013 IEEE. Personal use of this material is permitted. Permission from IEEE must be obtained for all other users, including reprinting/ republishing this material for advertising or promotional purposes, creating new collective works for resale or redistribution to servers or lists, or reuse of any copyrighted components of this work in other works.

(below follows the accepted version) 


\title{
Flexible fault current contribution with inverter interfaced distributed generation
}

\author{
Thomas Wijnhoven, GSM, IEEE, and Geert Deconinck, SM, IEEE \\ ELECTA, Department of Electrical Engineering, KU Leuven, Belgium
}

\begin{abstract}
This paper describes a control scheme to flexibly control the positive and negative sequence fault current contribution of inverter interfaced distributed generation (IIDG). This flexible control can be beneficial to limit the impact of distributed generation (DG) on existing grids. This way, a large scale integration of DG becomes possible in existing grids. A double synchronous reference frame (DSRF) control strategy is chosen. The phase locked loop (PLL) and current controller are based on available techniques and are described briefly. The performance of the control system is illustrated in a PSCAD simulation environment. Both symmetrical and asymmetrical faults are applied on a generic European medium voltage grid. Only the setpoints during faults have to be changed to obtain a specific fault response, creating the required flexibility.
\end{abstract}

Index Terms-distributed power generation, fault currents, inverter, power system protection

\section{INTRODUCTION}

Combined heat and power (CHP) units, wind turbines and solar installations are often installed near consumers and near open spaces, and their location is not determined by the presence of a suitable high voltage (HV) grid connection point. By integrating them in neighbouring distribution grids, a costly HV grid connection can be avoided and distributed generators (DG's) become more cost efficient. If large amounts of DG could be integrated in existing grids, this would enable a cost efficient (intermediate) step towards a lower carbon, more renewable power system for the 21 st century. In this paper, inverter interfaced distributed generators (IIDG's) are considered, as their power electronic interface allows a flexible control of the DG. More specifically, the reaction of IIDG's during grid faults is considered. It is shown that it is possible to control the positive and negative sequence fault current response within a time frame that is relevant for the protection system.

The paper is organised as follows: Section II describes phase locked loop (PLL) structures that are able to give an accurate estimation of positive and negative sequence angles. Both angles are required for the current controller in the double synchronous reference frame (DSRF) that is explained in section III. Finally, in section IV this control system is evaluated in simulations of a small medium voltage (MV) network to illustrate its performance and its capabilities.

T. Wijnhoven has a Ph. D. fellowship of the Research Foundation - Flanders (FWO) and wishes to acknowledge the financial support of the FWO.

E-mail contact: thomas.wijnhoven@esat.kuleuven.be

\section{PLL DURING FAULTS}

In general, the only grid information a DG unit has, is its local voltage and frequency. Only when the phase angles of the positive and negative sequence voltage at the point of common coupling (PCC) are known, a DG is able to inject a specific amount of active (in-phase) and reactive (quadrature) positive/negative sequence current. Since a DSRF controller will be used in section III, a PLL is required to track the phase angles.

During fault situations, positive, negative and zero sequence voltages appear. Using standard (positive sequence) PLL structures results in a bad estimation of the positive sequence angle [1]. Several methods to separate positive and negative sequence fundamental frequency components have been developed and are described in literature [2]-[5]. The positive sequence angle can then be determined from the positive sequence voltage with a standard PLL. The fundamental frequency negative sequence angle $\theta^{-}$is related to this positive sequence angle $\theta^{+}$by equation 1 , where $\gamma$ is constant in steady state. The zero sequence angle is usually not required, but can be determined with a standard PLL after using equation 2 to determine the zero sequence voltage.

$$
\begin{gathered}
\theta^{-}=-\theta^{+}+\gamma \\
u_{0}=\frac{1}{3}\left(u_{a}+u_{b}+u_{c}\right)
\end{gathered}
$$

The main idea of the different methods is to apply a filter to obtain the fundamental frequency components and a 90 degree phase shifted signal. Afterwards, the instantaneous symmetrical components transformation, as described in [6], can be applied. These methods differ in speed (with respect to changes) and in robustness (with respect to harmonic distortion). In this paper, the separation of positive and negative sequence components is made after the Clarke transformation $(a b c \rightarrow \alpha \beta)$, similar to [5]. Although more advanced filtering techniques have a better performance with distorted voltage signals, this simple delayed signal method was chosen in this paper because harmonic distortions only play a minor role in the simulations performed in section IV.

\section{Current controller}

After a PLL is constructed, the positive and negative sequence phase angles are known. As [7] pointed out, a DSRF controller is capable of controlling a voltage source converter's positive and negative sequence currents. In Fig. 1 the general structure of a DSRF current controller is shown. First, a 


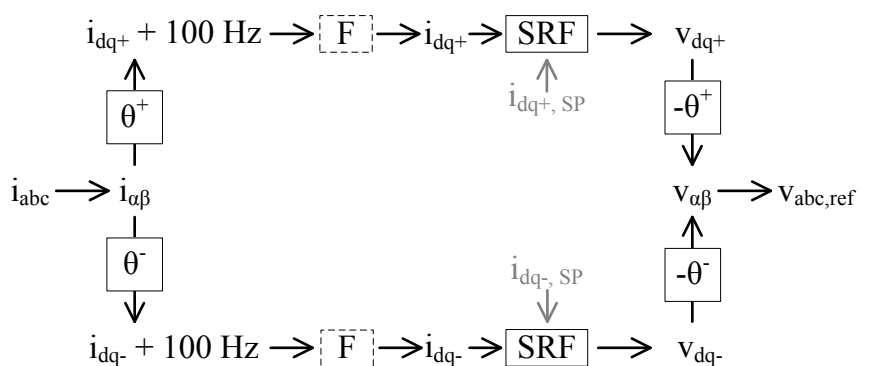

Fig. 1. General principle of a DSRF current controller

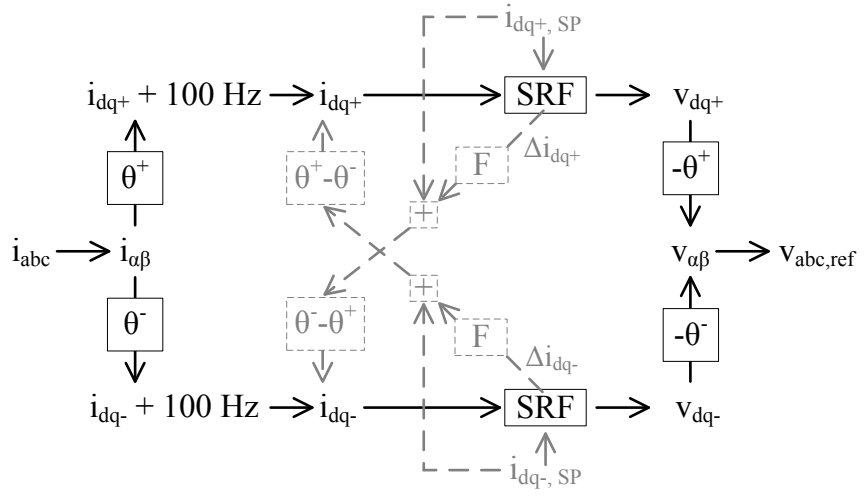

Fig. 2. Principle of the decoupled DSRF current controller

Clarke $(a b c \rightarrow \alpha \beta)$ transformation is applied. Afterwards, two synchronous reference frames are made by transforming these currents to the positive sequence synchronous reference frame (with angle $\theta^{+}$) and to the negative sequence synchronous reference frame (with angle $\theta^{-}$). This means that the total current is transformed to both reference frames. Therefore, there is a DC component, the positive sequence current, and a second harmonic component $(100 \mathrm{~Hz}$ for a nominal grid frequency of $50 \mathrm{~Hz}$ ) in the positive sequence reference frame. Likewise, the negative sequence frame contains a DC component, the negative sequence current, and a second harmonic component. By removing the second harmonic components with a band stop filter (see Fig. 1: "F"), a classic DC control system can be built to control the positive and negative sequence currents towards their setpoints $i_{\mathrm{dq}+, S P}$ and $i_{\mathrm{dq}-\mathrm{SP}}$ (see Fig. 1: "SRF"). This means that simple PI controllers can be used to avoid steady state errors. The reference signal for the converter, $\mathrm{v}_{\mathrm{abc}, \mathrm{ref}}$, is calculated by transforming the positive and negative sequence reference frames back to the stationary frame, adding both and applying the inverse Clarke transformation (see Fig. 1).

However, the band stop filters, that were used in [7] to suppress the positive sequence components in the negative sequence frame and vice versa, lead to phase delay and instability of the controller when a fast current control is required [8]. This results in a relatively slow current control, unsuitable for the purposes of fault current control within a few fundamental periods ( $\ll 100 \mathrm{~ms}$ ).

Reyes et al. [9] recently developed an interesting decoupled

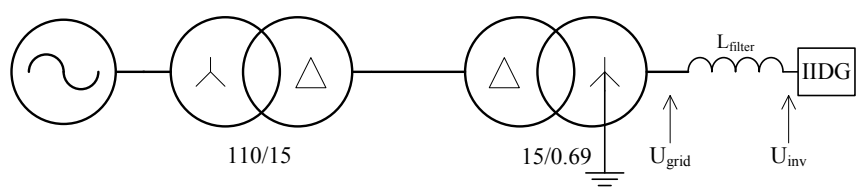

Fig. 3. Grid model

TABLE I

GRID DATA

\begin{tabular}{c|c|c}
\hline Element & Property & Value \\
\hline HV grid & Rated voltage (L-L) & $110 \mathrm{kV}$ \\
& Short-circuit power & $1200 \mathrm{MVA}$ \\
& X/R ratio & 10 \\
& Frequency & $50 \mathrm{~Hz}$ \\
\hline MV transformer & Rating & $40 \mathrm{MVA}$ \\
& Voltage ratio & $110 \mathrm{kV} / 15 \mathrm{kV}$ \\
& Connection & Yd \\
& Impedance voltage & $12 \%$ \\
& Copper losses & $0.5 \%$ \\
\hline LV transformer & Rating & $2 \mathrm{MVA}$ \\
& Voltage ratio & $15 \mathrm{kV} / 690 \mathrm{~V}$ \\
& Connection & Dy11 (solidly earthed) \\
& Impedance voltage & $6 \%$ \\
& Copper losses & $0.95 \%$ \\
\hline Inverter & Rating & $2000 \mathrm{kVA}$ \\
& DC voltage & $2650 \mathrm{~V}$ (constant) \\
\hline Filter & L & $1 \mathrm{mH}$ \\
& R & $3 \mathrm{~m} \Omega$
\end{tabular}

DSRF current controller. This controller no longer uses band stop filters, but instead uses a decoupling system to remove the second harmonic components in the positive and negative sequence control frames. This method was applied here and its principle is illustrated in Fig. 2. The feedback filters for the error signals $\Delta \mathrm{i}_{\mathrm{dq}+}$ and $\Delta \mathrm{i}_{\mathrm{dq}-}$ in the decoupling loop (see Fig. 2: "F"), were tuned to obtain a faster current control, even for a high power (2 MVA) IIDG. More details about the decoupled DSRF scheme are found in [9].

\section{ILLUSTRATION OF FLEXIBLE FAULT CURRENT CONTROL}

\section{A. Simulation model}

The simulation model is built in PSCAD. Fig. 3 shows the grid model that is used and table I summarizes the grid properties. The MV voltage level was chosen to be $15 \mathrm{kV}$. Any voltage around $10-20 \mathrm{kV}$ is realistic for European MV distribution networks: e.g. the CIGRE benchmark grid for integrating DG in MV distribution networks is based on a German MV grid and has a voltage level of $20 \mathrm{kV}$ [10], while other studies of European MV grids have lower voltage levels: $10 \mathrm{kV}$ in the Netherlands [11] and $11 \mathrm{kV}$ in the UK [12].

The IIDG is connected to $690 \mathrm{~V}$ and is modelled as an averaged value model (AVM) [3]. It uses the PLL and decoupled DSRF current controller that are described in the previous sections II and III. In situations without a negative 
sequence voltage, the inverse of the positive sequence phase angle is used for the negative sequence phase angle, though this approach is not correct if the goal is to control the inphase and quadrature negative sequence currents individually. Therefore, the negative sequence phase angle is tracked by the PLL when there is a negative sequence voltage.

As an additional simplification the DC bus voltage is kept constant. This allows evaluating the performance of the decoupled DSRF current controller without considering the impact of the DC bus voltage in the simulations. This DC bus voltage is chosen relatively high because of two reasons:

1) Pulse width modulation is chosen (as opposed to space vector modulation)

2) The IIDG must be capable of delivering $100 \%$ reactive current (see equation 3, with $U_{\text {inv }}$ and $U_{\text {grid }}$ as indicated in Fig. 3 and $U_{\mathrm{dc}}$ the required DC bus voltage)

$$
\begin{aligned}
U_{i n v} & =I_{i n v} \cdot Z_{f i l t e r}+U_{\text {grid }} \\
& =1674 \cdot e^{-j \frac{\pi}{2}} \cdot(0.0030+j 0.3142)+\frac{690}{\sqrt{3}} \\
\Rightarrow U_{d c} & =\left|U_{i n v}\right| \cdot \sqrt{2} \cdot 2=2614 \mathrm{~V}
\end{aligned}
$$

\section{B. Two phase fault at $L V$}

In all simulations, the IIDG is delivering half of its rated current with a power factor equal to 0.95 before the fault $(\mathrm{P}=0.95 \mathrm{MW}, \mathrm{Q}=0.31 \mathrm{MVAr})$. Then, in the first simulation, an ideal two phase fault $\left(\mathrm{R}_{\text {fault }} \approx 0 \Omega\right)$ is applied at the low voltage (LV) side of the step-up transformer at $t=5 \mathrm{~s}$ and the fault is removed at $t=5.2 \mathrm{~s}$. Only the filter impedance is between the fault and the IIDG.

To illustrate that the IIDG can react in a random way, three different current setpoint strategies are applied. All strategies change the current setpoints during the fault, based on the positive and negative sequence voltage measured by the PLL.

In strategy A (Fig. 4), only positive sequence currents are injected (maximal reactive current). Fig. 4a-4b show the grid voltages at $\mathrm{LV}$ and MV before and during the fault. Fig. 4c shows the voltages as measured by the PLL (positive and negative sequence voltage are equal at the location of the fault) and Fig. 4d gives the setpoints of the current controller before, during and after the fault. Fig. 4e then gives the inverter output current. As can be seen, both the settling time at the start $(t=5 s)$ and at the end $(t=5.2 s)$ of the fault are very fast. The PLL voltages have a fast settling time, but exhibit some transients.

In strategy B (Fig. 5), other setpoints are chosen during the fault: the negative sequence quadrature current is related to the negative sequence voltage according to equation 4 and the positive sequence in-phase current is reduced during faults.

$$
i_{q-}=\frac{u_{d-}}{u_{d+}} \cdot i_{q+}
$$

In strategy $\mathrm{C}$ (Fig. 6), the current setpoints are unaltered during the fault. For both strategy $\mathrm{B}$ and $\mathrm{C}$, the voltage during the fault is not shown as it is very similar to strategy A. This is

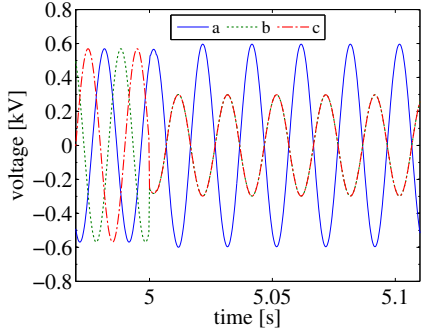

(a)

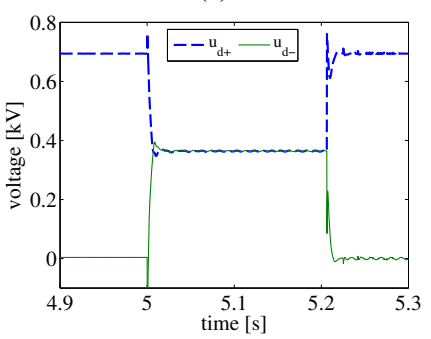

(c)

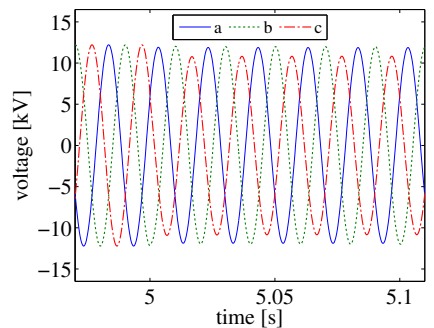

(b)

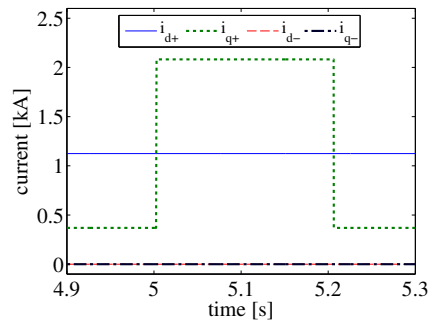

(d)

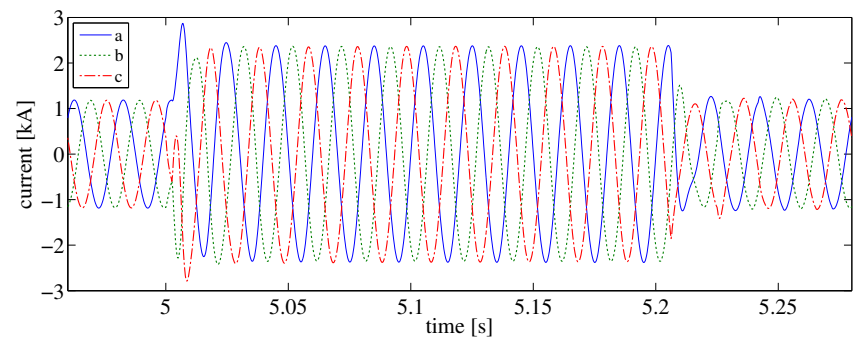

(e)

Fig. 4. Two phase fault at LV, strategy A (no negative sequence current injection): (a) Voltage at the LV side and (b) at the MV side of the stepup transformer. (c) Positive and negative sequence voltage as measured by the PLL. (d) Setpoints (in the synchronous reference frames) of all sequence components. (e) Inverter output current at LV (before the step-up transformer).

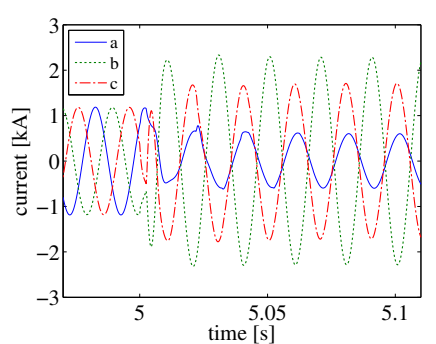

(a)

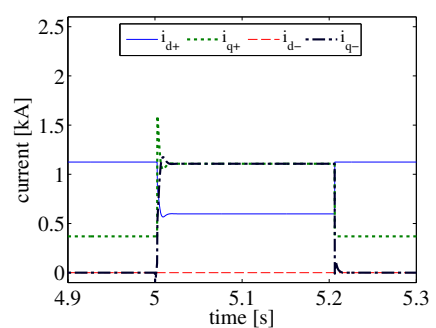

(b)
Fig. 5. Two phase fault at LV, strategy B (with negative sequence current injection): (a) Inverter output current at LV. (b) Setpoints (in the synchronous reference frames) of all sequence components.

due to the limited short-circuit power of the IIDG compared to the grid. This topic will be addressed further in section IV-F.

The fault current responses and the setpoints that are applied (see Fig. 5 and Fig. 6) illustrate that a totally different fault current response, with a fast settling time, is obtained by applying different setpoints. This means that the fault behaviour can be changed in a flexible way according to the grid requirements. 


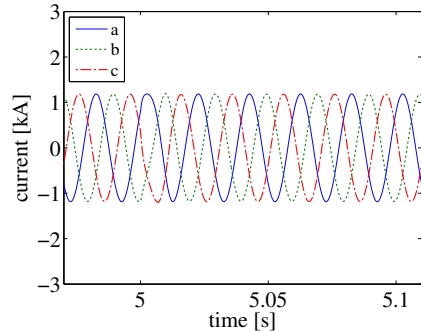

(a)

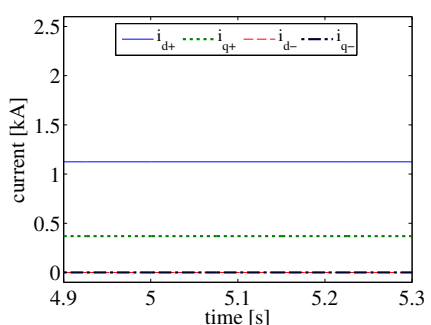

(b)
Fig. 6. Two phase fault at LV, strategy $\mathrm{C}$ (no change in current setpoints): (a) Inverter output current at LV. (b) Setpoints (in the synchronous reference frames) of all sequence components.

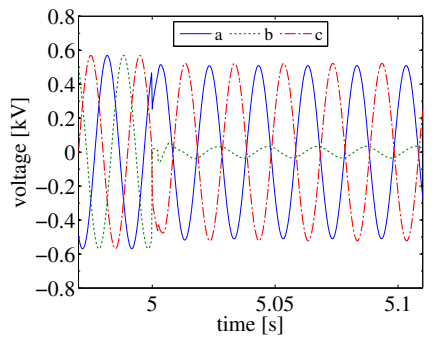

(a)

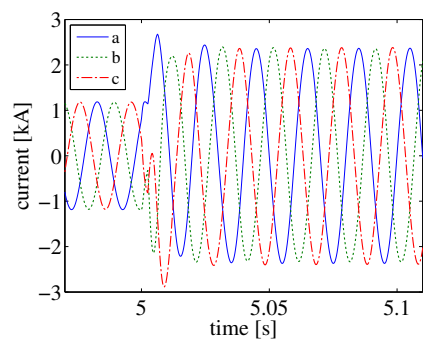

(c)

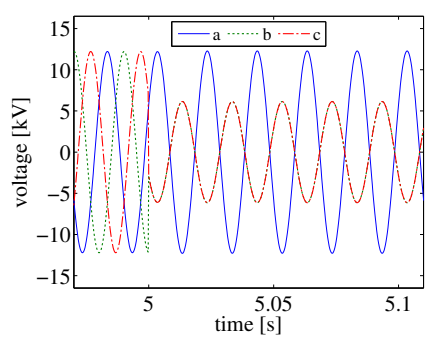

(b)

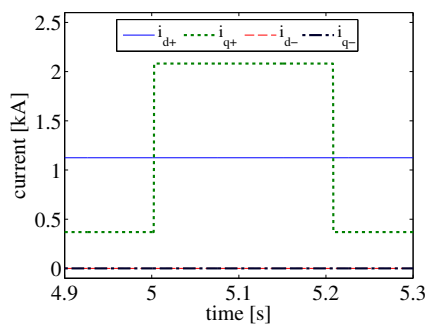

(d)
Fig. 7. Two phase fault at MV, strategy A (no negative sequence current injection): (a) Voltage at the LV side and (b) at the MV side of the step-up transformer. (c) Inverter output current at LV. (d) Setpoints (in the synchronous reference frames) of all sequence components.

\section{Two phase fault at $M V$}

In the next simulation, an ideal two phase fault $\left(\mathrm{R}_{\text {fault }} \approx 0 \Omega\right)$ is applied at the MV side of the step-up transformer. The same setpoint strategies $\mathrm{A}$ and $\mathrm{B}$ are used here and the results are given in Fig. 7 (strategy A) and Fig. 8 (strategy B). Again it is shown that just by applying a different setpoint strategy during the fault, a totally different fault response is obtained.

\section{Three phase fault at $L V$}

The results of the simulation for a three phase fault at the LV side of the step-up transformer are not shown in this paper. In this case, the voltage at the IIDG is about zero and the PLL is unable to track the phase angles. Because most faults at the LV side of the step-up transformer are permanent, these faults will usually require a disconnection of the IIDG from the grid. In other cases, the IIDG's inverter should be blocked and kept off-line to restart as soon as the voltage is normalised again.

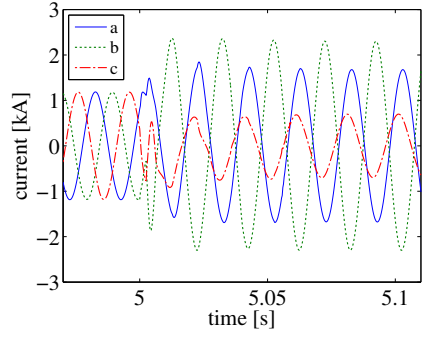

(a)

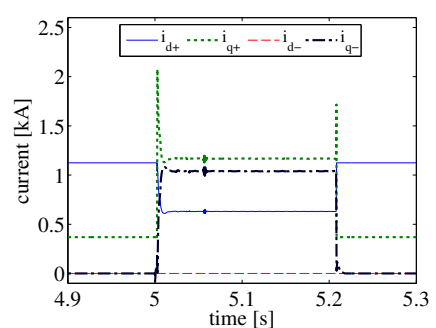

(b)
Fig. 8. Two phase fault at MV, strategy B (with negative sequence current injection): (a) Inverter output current at LV. (b) Setpoints (in the synchronous reference frames) of all sequence components.

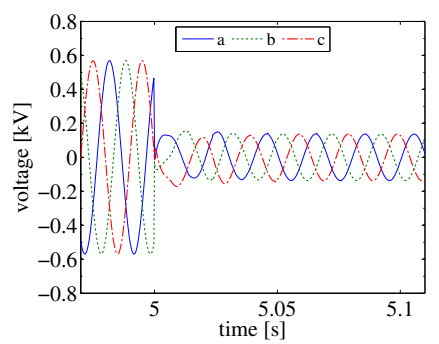

(a)

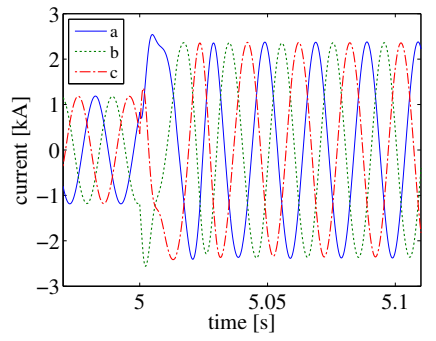

(c)

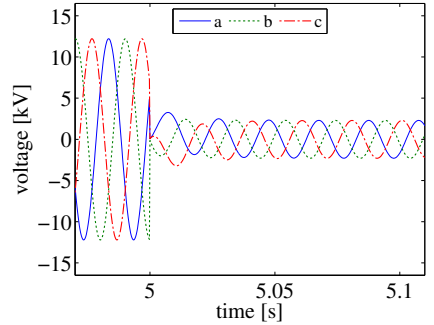

(b)

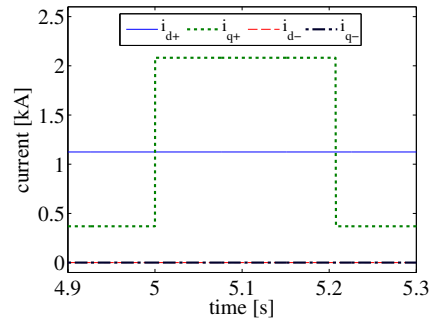

(d)
Fig. 9. Three phase fault at MV, strategy A (no negative sequence current injection): (a) Voltage at the LV side and (b) at the MV side of the step-up transformer. (c) Inverter output current at LV. (d) Setpoints (in the synchronous reference frames) of all sequence components.

\section{E. Three phase fault at $M V$}

Next, a three phase fault is applied at the MV level $\left(\mathrm{R}_{\text {fault }}=0.5 \Omega\right)$. In this fault case, there is no negative sequence voltage. Two different strategies are shown: strategy A (Fig. 9) is the same as in the previous cases, but strategy B (Fig. 10) is altered to illustrate the flexibility of the control scheme. Here, the injected quadrature negative sequence current during a fault is set to be the same as the quadrature positive sequence current.

In these simulations, the PLL settling time is a bit larger, which is noticeable in the injected currents of both Fig. 9c and Fig. 10a. The fault response of the latter (strategy B) is completely different from the former (strategy A), again demonstrating the flexibility of the control scheme.

\section{F. Remark on fault contributions}

The simulations show the performance of the current controller and the flexible fault current contribution of the IIDG. On the MV grid, the impact of one 2 MVA IIDG unit is very 


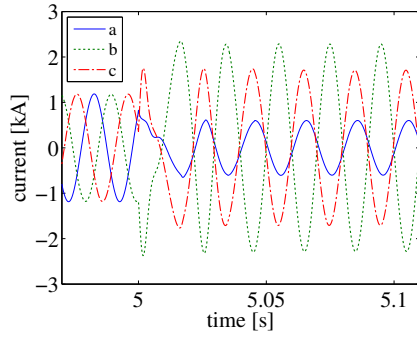

(a)

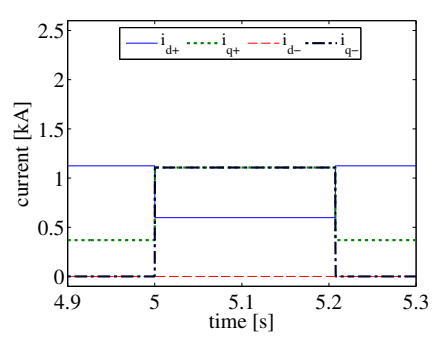

(b)
Fig. 10. Three phase fault at MV, strategy B (with negative sequence current injection): (a) Inverter output current at LV. (b) Setpoints (in the synchronous reference frames) of all sequence components.

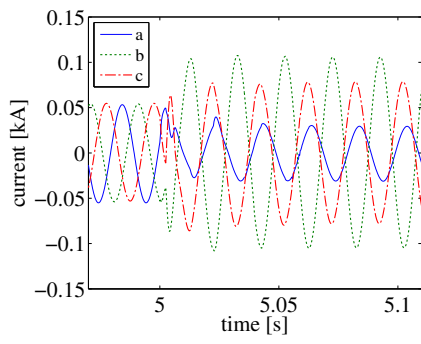

(a)

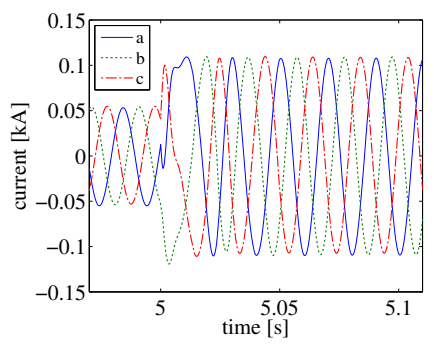

(b)
Fig. 11. Examples of the fault current responses on MV: (a) MV fault current contribution of the IIDG for a two phase fault at MV (strategy B) and (b) for a three phase fault at MV (strategy A).

limited, as it can only deliver about 77 A without overloading the IGBT's of the inverter. Fig. 11 shows two examples of the limited MV current contribution of the IIDG during a MV fault. The simulated MV grid has short-circuit levels of about $10 \mathrm{kA}$. Therefore, the current contribution of IIDG can be considered as not important at today's low integration levels, which is in line with the conclusions of [12]. In future grids, with high amounts of DG's in the MV grid, these conclusions have to be verified. The impact of the IIDG will also increase if the short-circuit levels of the MV grid are reduced.

As was indicated in the introduction, the settling time of the current controller is well below $100 \mathrm{~ms}(5$ cycles at $50 \mathrm{~Hz})$. For all protections that do not decide immediately $(<20 \mathrm{~ms})$, this is relevant and thus the flexible control is relevant for the protection system.

The limited fault current contribution can also be seen as an advantage. Whenever standard DG units (with synchronous or (doubly fed) induction generators) pose integration challenges to the MV grid or when they are unable to comply with fault ride-through (FRT) requirements, IIDG are likely to provide a solution. A full evaluation of the impact of the different strategies is outside the scope of this paper and is part of future research.

\section{COnClusions}

This paper describes a current controller for a flexible fault current contribution of IIDG. It was shown that, when the phase angles are tracked with an adequate PLL, a decoupled DSRF current controller is capable of flexibly controlling both positive and negative sequence current injections.
Several simulations were performed in PSCAD to illustrate the performance of the current controller. It was seen that the contribution of IIDG to the fault currents in a MV grid is very limited. Even with a considerable amount of IIDG, protection problems seem unlikely. Future work will focus on solutions this flexible control of IIDG fault current contributions can bring to increase the amount of DG that can be integrated in MV distribution grids.

\section{ACKNOWLEDGEMENTS}

The authors would like to thank Agusti Egea Alvarez from Citcea UPC, Barcelona, Spain, who helped implementing the simplified positive/negative sequence PLL filter during his research stay at Electa, KU Leuven.

\section{REFERENCES}

[1] P. Rodriguez, J. Pou, J. Bergas, J. I. Candela, R. P. Burgos, and D. Boroyevich, "Decoupled Double Synchronous Reference Frame PLL for Power Converters Control,' IEEE Transactions on Power Electronics, vol. 22, no. 2, pp. 584-592, Mar. 2007.

[2] G. Saccomando and J. Svensson, "Transient operation of grid-connected voltage source converter under unbalanced voltage conditions," in IEEE 36th Industry Applications Society (IAS) Annual Meeting, vol. 4, 2001, pp. 2419-2424.

[3] T. Wijnhoven, J. Tant, and G. Deconinck, "Inverter Modelling Techniques for Protection Studies," in 3rd IEEE International Symposium on Power Electronics for Distributed Generation Systems (PEDG), Aalborg, Denmark, Jun. 2012, pp. 187-194.

[4] P. Rodriguez, A. Luna, M. Ciobotaru, R. Teodorescu, and F. Blaabjerg, "Advanced Grid Synchronization System for Power Converters under Unbalanced and Distorted Operating Conditions," in IEEE 32nd Annual Conference on Industrial Electronics (IECON), Nov. 2006, pp. 5173-5178.

[5] J. Svensson, M. Bongiorno, and A. Sannino, "Practical Implementation of Delayed Signal Cancellation Method for Phase-Sequence Separation," IEEE Transactions on Power Delivery, vol. 22, no. 1, pp. 18-26, Jan. 2007.

[6] M. Iravani and M. Karimi-Ghartemani, "Online estimation of steady state and instantaneous symmetrical components," IEE Proceedings - Generation, Transmission and Distribution, vol. 150, no. 5, pp. 616-622, 2003.

[7] H.-S. Song and K. Nam, "Dual current control scheme for PWM converter under unbalanced input voltage conditions," IEEE Transactions on Industrial Electronics, vol. 46, no. 5, pp. 953-959, 1999.

[8] F. D. Freijedo, A. G. Yepes, O. López, A. Vidal, and J. Doval-Gandoy, "Three-Phase PLLs With Fast Postfault Retracking and Steady-State Rejection of Voltage Unbalance and Harmonics by Means of Lead Compensation," IEEE Transactions on Power Electronics, vol. 26, no. 1, pp. 85-97, Jan. 2011.

[9] M. Reyes, P. Rodriguez, S. Vazquez, A. Luna, R. Teodorescu, and J. M. Carrasco, "Enhanced Decoupled Double Synchronous Reference Frame Current Controller for Unbalanced Grid-Voltage Conditions," IEEE Transactions on Power Electronics, vol. 27, no. 9, pp. 3934-3943, Sep. 2012.

[10] K. Rudion, A. Orths, Z. Styczynski, and K. Strunz, "Design of benchmark of medium voltage distribution network for investigation of DG integration," in IEEE Power Engineering Society General Meeting, 2006, p. 6.

[11] E. J. Coster, J. Morren, J. Myrzik, and W. Kling, "Comparison of MV-grid structures on fault ride through behavior of MV-connected DG-units," in CIRED 20th International Conference on Electricity Distribution, Prague, 2009, p. 4.

[12] K. Jennett, F. Coffele, and C. Booth, "Comprehensive and quantitative analysis of protection problems associated with increasing penetration of inverter-interfaced DG," in 11th IET International Conference on Developments in Power Systems Protection (DPSP 2012), 2012, p. 6. 